\title{
Beta-Trace Protein as a Potential Marker of Acute Kidney Injury: A Pilot Study
}

\author{
Katrien Leyssens ${ }^{a} \quad$ Niels Van Regenmortel ${ }^{b} \quad$ Ella Roelant $^{c} \quad$ Khadija Guerti $^{d}$ \\ Marie Madeleine Couttenye ${ }^{\mathrm{a}, \mathrm{g}}$ Philippe G. Jorens ${ }^{\mathrm{e}}$ Walter Verbrugghe ${ }^{\mathrm{e}}$ \\ Amaryllis H. Van Craenenbroeck ${ }^{\mathrm{f}} \mathrm{g}$ \\ aDepartment of Nephrology and Hypertension, Antwerp University Hospital, Edegem, Belgium; bepartment \\ of Intensive Care Medicine, ZNA Stuivenberg, Antwerp, Belgium; ' ${ }^{C}$ linical Trial Center (CTC), Antwerp University \\ Hospital, Edegem, Belgium; 'Department of Clinical Chemistry, Antwerp University Hospital, Edegem, Belgium; \\ eDepartment of Intensive Care Medicine, Antwerp University Hospital, Edegem, Belgium; fDepartment of \\ Nephrology and Renal Transplantation, University Hospital Leuven, Leuven, Belgium; 9Laboratory of Experimental \\ Medicine and Pediatrics, University of Antwerp, Antwerp, Belgium
}

\section{Keywords}

Acute kidney injury - Beta-trace protein - Intensive care unit - Neutrophil gelatinase-associated lipocalin · Cystatin C

\begin{abstract}
Introduction: Acute kidney injury (AKI) is a frequent complication among patients in the intensive care unit (ICU). The limitations of serum $\mathrm{Cr}(\mathrm{s} C r)$ in timely detecting $\mathrm{AKI}$ are well known. Beta-trace protein (BTP) is emerging as a novel endogenous glomerular filtration rate marker. The aim of this study was to explore the role of BTP as a marker of AKI. Methods: Patients admitted to the ICU undergoing surgery were included. BTP, sCr, Cystatin C (CysC), and neutrophil gelatinase-associated lipocalin (NGAL) were measured preoperatively, postoperatively (post-op), and at the first (D1) and second (D2) post-op day. AKI was defined as an increase of $\mathrm{sCr}$ to $\geq 1.5$-fold from baseline within 2 days after surgery. $\boldsymbol{R e}$ sults: Of the 52 patients studied, 10 patients (19\%) developed AKI. Patients with AKI were older (69.6 \pm 10.7 vs. $58.1 \pm$ 16.7 years, $p=0.043$ ) and had a longer length of ICU stay (13 [IQR 6-49] vs. 6 [IQR 5-8] days, $p=0.032$ ). Between the 2
\end{abstract}

karger@karger.com www.karger.com/kbr

Karger $\stackrel{\text { ' }}{5}$

BOPEN ACCESS
(C) 2021 The Author(s).

Published by S. Karger AG, Basel

This article is licensed under the Creative Commons AttributionNonCommercial-NoDerivatives 4.0 International License (CC BYNC-ND) (http://www.karger.com/Services/OpenAccessLicense). Usage and distribution for commercial purposes as well as any distribution of modified material requires written permission. groups, the evolution of $\mathrm{BTP}, \mathrm{sCr}$, CysC, and NGAL over time differed significantly, with overall higher values in the AKI group. ROC analysis for the detection of AKI within 2 days after surgery showed a great accuracy for BTP. The area under the curve (AUC) for BTP post-op; D1; and D2 was, respectively, $0.869 \pm 0.049 ; 0.938 \pm 0.035$; and $0.943 \pm 0.032$. The discriminative power of a BTP measurement on D1 was superior in detecting AKI compared to NGAL (adjusted $p$ val$\mathrm{ue}=0.027$ ). We could not detect a significant difference between the AUCs of other biomarkers (NGAL, $\mathrm{SCr}$, and CysC). Conclusion: Serum BTP is a promising marker for diagnosing AKI in ICU patients undergoing surgery.

$$
\begin{aligned}
& \text { () } 2021 \text { The Author(s). } \\
& \text { Published by S. Karger AG, Basel }
\end{aligned}
$$

\section{Introduction}

Acute kidney injury (AKI) is common in patients at the intensive care unit (ICU), with incidences varying from 35 up to $48 \%$ after surgery, especially after cardiac surgery [1-4]. Epidemiological data suggest that an epi- 
sode of AKI increases mortality risk, both at the long and short term [1-12], as well as the risk for developing CKD $[3,4,13]$.

The limitations of serum $\mathrm{Cr}(\mathrm{sCr})$ in diagnosing AKI are well recognized, as it varies with gender, age, muscle mass, dietary intake, and hydration status [14]. Furthermore, after an injurious event, the rise in $\mathrm{sCr}$ is slow (12$24 \mathrm{~h}$ ) due to increased tubular excretion when the glomerular function decreases $[1,13,15]$. This concept is in contrast with the currently pursued principle of an early goal-directed therapy. The diagnosis of AKI is challenging and follows international, Cr-based criteria such as the risk, injury, failure, loss of kidney function, and endstage kidney disease (RIFLE) criteria, the AKI network (AKIN) criteria, and the Kidney Disease: Improving Global Outcomes Foundation (KDIGO) [1] .

Given the potential pitfalls of Cr-based definitions, an increasing number of "novel" renal injury biomarkers have been investigated during the last decades. The most studied biomarker is neutrophil gelatinase-associated lipocalin (NGAL), also called lipocalin-2 [16, 17]. The molecule is freely filtered through the glomerulus and almost completely reabsorbed by endocytosis in the proximal tubule. As such, serum NGAL is regarded as an early marker for tubular injury. It can be identified in plasma and urine starting $2-4 \mathrm{~h}$ after kidney injury and precedes an increase in sCr by $12-24 \mathrm{~h}[5,7,8,11-14,17-20]$. However, elevated plasma NGAL levels are not specific, and increased plasma levels are also reported in malignancy, $\mathrm{CKD}$, pancreatitis, chronic obstructive pulmonary disease, and urinary tract infection. Moreover, levels of NGAL depend on gender, age, liver function, and inflammatory parameters $[1,11,14,18]$.

Cystatin $\mathrm{C}(\mathrm{CysC})$ is a low-molecular-weight-protein $(12 \mathrm{kDa})$ that is produced in all nucleated body cells, at a constant rate. It is freely filtered through the glomerulus with complete tubular reabsorption and catabolization, no reabsorption into the bloodstream, and no renal tubular secretion. As such, CysC is regarded as a good filtration marker [21]. CysC has been reported to rise faster than sCr after AKI $[22,23]$ and GFR using CysC-based equations (or CysC combined with sCr-based equations) have been demonstrated to be superior over Cr-based equations. As $\mathrm{Cys} C$ is less influenced by muscular mass and gender, there are reports demonstrating that $\mathrm{CysC}$ is influenced by smoking, high-dose steroid therapy, obesity, hyperthyroidism, inflammation, and malignancy [23]. Furthermore, there are concerns related to the lack of standardization in CysC measurements [23].
Beta-trace protein (BTP), also called prostaglandin D2 synthase, is another novel endogenous GFR marker [19, 24-27]. This low-molecular-weight-protein (23-29 kDa) appears to be freely filtered through the glomerulus with minimal nonrenal elimination (whether or not this is followed by tubular reabsorption and degradation requires further study $[24,28])$. The main source of BTP is the central nervous system, as the molecule is generated at a constant rate by glial cells [29]. According to the literature, normal serum BTP levels in men range from 0.37 to $0.77 \mathrm{mg} / \mathrm{L}$ and in women $0.40-0.70 \mathrm{mg} / \mathrm{L}[24,30]$. During the last decade, several BTP-based GFR equations were proposed to translate plasma BTP levels into estimated GFR [25, 31-33]. In some studies, BTP appeared to be a more sensitive indicator of GFR than $\mathrm{sCr}$, and the molecule was put forward as an indicator of reduced GFR, particularly in the Cr-blind range [19, 25-27]. The estimated half-life of BTP is approximately $1.2 \mathrm{~h}$, which is shorter than that of $\mathrm{sCr}(3.8 \mathrm{~h})$ [21]. Moreover, no technical drawbacks are present as the nephelometric analysis is technically easy and rather inexpensive. Whereas the relationship between BTP and CKD is well established [19, 24, 30, 34-36], little is known about BTP in AKI. Experimental data in a murine model of immune-mediated nephritis demonstrate an increase in both urinary and serum BTP, reaching their peak levels on day 14 [37]. Furthermore, a prospective trial in patients after cardiac surgery demonstrated that serum BTP levels preoperatively (pre-op) predicted AKI [22]. This pilot study aims to explore the potential role of serum BTP as a reliable marker of AKI in ICU patients undergoing surgery, compared to sCr, serum CysC, and serum NGAL.

\section{Materials and Methods}

\section{Subjects}

This prospective observational study, using spare serum samples of included patients, was conducted in the 45-bed ICU of the Antwerp University Hospital from March 2017 until February 2018. Patients who were admitted to the ICU, for more than 3 days, who had spare serum samples left and who underwent surgery during their observation, were screened on a weekly basis for inclusion. Surgery could not include hepatic- or neurosurgery as BTP is produced by the central nervous system and metabolized by the liver. Exclusion criteria were age $<18$ years, pre-op kidney dysfunction (eGFR CKD-EPI $<60 \mathrm{~mL} / \mathrm{min} / 1.73 \mathrm{~m}^{2}$ ), liver failure, pregnancy, and acute central neurological disorders. Also, patients with renal replacement therapy pre-op or within 2 days after surgery were excluded since all biomarkers are potentially influenced by this treatment. Patients were identified as medical or surgical patients, depending on their admission reason to the ICU, that is, for internal medicine or surgery. 


\section{Procedures}

Blood samples were collected at 4-time points: pre-op, postoperatively (post-op; first blood sample after surgery), and in the morning of the first (D1) and second (D2) post-op day. Baseline sCr was extracted from the medical files in retrospect and defined as the lowest stable value during the 3 months preceding surgery. If not available, the admission value was used as a surrogate baseline.

Upon collection, samples were centrifuged at 3,720 rpm for 10 min. Serum samples were stored at $4^{\circ} \mathrm{C}$ (maximum 4 days), transferred to $-20^{\circ} \mathrm{C}$ (maximum 7 days) and eventually at $-80^{\circ} \mathrm{C}$ until batch analysis. BTP, CysC, and NGAL were both measured using a nephelometric assay (BN II nephelometer Siemens) according to the manufacturer's protocols. Interassay coefficient of variation for BTP measurements was 7.8\%. Reference range for BTP for this technique is reported as $0-0.70 \mathrm{mg} / \mathrm{L}$. Interassay coefficient of variation for serum CysC measurements was $2.6 \%$, with a reference range for $\mathrm{CysC}$ of $0.62-1.11 \mathrm{mg} / \mathrm{L}$. sCr was measured using a photometric test, Dimension Vista 1500 system (Siemens).

Demographic parameters such as age, gender, BMI, and comorbidities such as diabetes mellitus, arterial hypertension, and thyroid disorders were extracted from the medical files. Furthermore, the occurrence of infection at admission, the use of corticosteroids, type of surgery and urgency, length of ICU stay, ICU mortality, and vasopressor therapy were obtained by data extraction from the ICU patient data management system (Metavision, iMDsoft, Düsseldorf, Germany). As outcome predictor scores, both the sequential organ failure assessment score (SOFA [38], a validated scoring for organ failure) and APACHE IV (a scoring for severity of illness [39]) were calculated. SOFA score was calculated using the worst values of the first $24 \mathrm{~h}$ of admission. APACHE IV score was calculated at admission.

For this study, AKI was defined as an increase of SCr to $\geq 1.5$ fold from baseline within 2 days after surgery. For staging of AKI, AKIN sCr criteria were used [10]. Stage 1 is defined as an increase of sCr to $\geq 1.5$-fold from baseline; stage 2 as an increase to $\geq 2$-fold; and stage 3 as an increase to $\geq 3$-fold from baseline sCr.

\section{Statistical Analysis}

To compare continuous variables between 2 groups, an independent samples $t$ test was used in case of normality and a MannWhitney U test otherwise. For categorical variables, Fisher's exact test was used because of low numbers. ROC analysis was performed to further characterize the diagnostic performance of NGAL, BTP, CysC, and sCr to detect the occurrence of AKI by D2. DeLong's method was used to compare the area under the correlated ROC's. As the 4 markers were compared 2 by 2, per time point $p$ values were adjusted for multiple testing using the Holm correction. For the correlation between 2 pre-op variables, Spearman rank correlation was calculated. Statistical analysis was performed using R 3.5.2 (R Foundation for Statistical Computing, 2018) and SAS 9.4 (SAS Institute Inc., Cary, NC, USA).

To compare the evolution over time of BTP between AKI and no-AKI group a linear mixed model was fitted using time (as a categorical variable), group (AKI or no-AKI), and the interaction between time and group as fixed effects and subject as a random effect. As there was a significant interaction between time and group, we looked at the difference in mean BTP per measured time point between the AKI and no-AKI groups. We also compared the preop BTP value to the post-op, D1, and D2 BTP value within the AKI and no-AKI group, respectively. $p$ values were adjusted for multiple testing using the Holm correction. The same was done for sCr, CysC, and NGAL. For all markers, a logarithmic transformation was considered to improve model assumptions. Statistical significance was set at 2 -tailed $p$ values $<0.05$.

\section{Results}

\section{Patients' Characteristics}

Out of 85 patients screened, 52 patients were included in the study (for type of surgery, see online suppl. Table 1 ; see www.karger.com/doi/10.1159/000514173 for all online suppl. material). From 10 out of 52 studied patients, baseline SCr was not available and the admission value was used as a surrogate baseline. Median time between the end of the surgery and the post-op sampling was 56 min (IQR: $47 \mathrm{~min}-1 \mathrm{~h} 14 \mathrm{~min}$ ), between the end of the surgery and the D1 samples was $12 \mathrm{~h} 32 \mathrm{~min}$ (IQR: $10 \mathrm{~h} 1 \mathrm{~min}-15 \mathrm{~h} 43 \mathrm{~min}$ ), and between the end of surgery and the D2 samples was $35 \mathrm{~h} 40 \mathrm{~min}$ (IQR: $33 \mathrm{~h} 22 \mathrm{~min}-$ 39 h $57 \mathrm{~min})$.

Of the 52 patients studied, 10 patients (19\%) developed AKI by day 2. In 8 patients, this was due to acute tubulus necrosis secondary to shock (septic, hemorrhagic, obstructive, or cardiogenic shock). In 2 patients, this was prerenal and quickly resolved (within 2 days) with administration of intravenous fluids. All these renal diagnoses were clinically made as in none of these patients a renal biopsy was performed. Five of the AKI patients reached AKI stage 1, 4 patients AKI stage 2, and 1 patient developed AKI stage 3. Baseline characteristics of the patients with AKI were compared with subjects who did not develop AKI (Table 1). Patients with AKI were older and had a longer length of ICU stay than patients without AKI. There were no statistically significant differences with respect to sex, BMI, baseline sCr, SOFA score, or APACHE IV score. Furthermore, no statistical significant difference was found between the 2 groups regarding the use of vasopressors, type of patients (medical/surgical), and urgency of surgery. Pre-op, higher serum BTP levels, and serum CysC levels were observed in the AKI group versus non-AKI group (Table 1).

\section{Beta-Trace Protein, Neutrophil Gelatinase-Associated Lipocalin, Cystatin C, and Serum Cr: Evolution Over Time \\ Graphical representation of the kinetics of the serum} levels of BTP, NGAL, CysC, and Cr is shown in Figure 1. The evolution over time of all 4 markers differed signifi- 
Table 1. Patients' characteristics and clinical outcomes

\begin{tabular}{|c|c|c|c|}
\hline & AKI, $n=10$ & No-AKI, $n=42$ & $p$ value \\
\hline \multicolumn{4}{|l|}{ Patients' characteristics } \\
\hline Age, years & $69.60(10.71)$ & $58.05(16.72)$ & 0.043 \\
\hline Sex (\% female) & $1(10 \%)$ & $14(33 \%)$ & 0.247 \\
\hline $\mathrm{BMI}, \mathrm{kg} / \mathrm{m}^{2}$ & $25.09(4.06)$ & $24.91(5.42)$ & 0.922 \\
\hline Baseline Cr, mg/dL & $0.88(0.14)$ & $0.78(0.20)$ & 0.082 \\
\hline \multicolumn{4}{|l|}{ Comorbidities, $n(\%)$} \\
\hline Diabetes mellitus & $3(30)$ & $6(14)$ & 0.349 \\
\hline Arterial hypertension & $6(60)$ & $24(57)$ & 1 \\
\hline Thyroid disorders & $1(13)(n=8)$ & $6(18)(n=33)$ & 1 \\
\hline Use of corticosteroids & $5(50)$ & $8(19)$ & 0.097 \\
\hline \multicolumn{4}{|l|}{ Admission } \\
\hline SOFA score at admission & $10(8.75-11.75)$ & $7(4-9)$ & 0.051 \\
\hline APACHE IV score at admission & $120.9(31.40)$ & $107.83(32.23)$ & 0.253 \\
\hline Use of vasopressor(s) during admission, $n(\%)$ & $10(100)$ & $33(79)$ & 0.178 \\
\hline Infection at admission, $n(\%)$ & $6(60)$ & $13(31)$ & 0.142 \\
\hline \multicolumn{4}{|l|}{ Outcome } \\
\hline ICU length of stay, days & $13(6.25-49)$ & $6(5-8)$ & 0.032 \\
\hline ICU mortality, $n(\%)$ & $2(20.00)$ & $4(9.52)$ & 0.325 \\
\hline \multicolumn{4}{|l|}{ Patients group, $n(\%)$} \\
\hline Internal medicine & $1(10.00)$ & $6(14.29)$ & 1 \\
\hline Surgical urgent & $3(30.00)$ & $14(33.33)$ & \\
\hline Surgical elective & $6(60.00)$ & $22(52.38)$ & \\
\hline \multicolumn{4}{|l|}{ Pre-op values } \\
\hline $\mathrm{SCr}, \mathrm{mg} / \mathrm{dL}$ & $0.98(0.18)$ & $0.79(0.23)$ & 0.171 \\
\hline Serum BTP, mg/L & $0.90(0.80-0.95)$ & $0.68(0.55-0.80)$ & 0.014 \\
\hline Serum NGAL, ng/mL & $111.45(74.38-168.50)$ & $99.35(65.60-126.50)$ & 0.252 \\
\hline Serum CysC, mg/L & $1.43(0.47)$ & $1.01(0.27)$ & 0.027 \\
\hline \multicolumn{4}{|c|}{$\begin{array}{l}\text { Variables are given as mean (SD) or median (interquartile range) in case of skewness. } p \text { values were calculated } \\
\text { using independent samples } t \text { test or Mann-Whitney U test; for categorical variables, Fisher's exact test was used. } \\
\text { Statistically significant values are reported in bold. AKI, acute kidney injury; No-AKI, no acute kidney injury; } \\
\text { ICU, intensive care unit; BTP, beta-trace protein; CysC, cystatin C; SCr, serum Cr. }\end{array}$} \\
\hline
\end{tabular}

cantly between the AKI and no-AKI group ( $p$ value for interaction between time and group for $\mathrm{BTP}<0.0001$, CysC $<0.0001$, NGAL $=0.011$, and $\mathrm{sCr}<0.0001)$. Estimates from these mixed models are used for betweengroup (at each time point groups were compared) and within-group comparisons (comparing pre-op vs. postop/D1/D2 values) (see online suppl. Tables 2, 3).

Altogether, patients in the AKI group had higher serum BTP values for every time point compared to the noAKI group (pre-op $p=0.0137$, all later times $p=0.0002$ or lower). BTP levels increased gradually over time in the AKI group, reaching a statistically significant increase at day 2 compared to the pre-op values $(p=0.004)$. In contrast, no increase was seen in the no-AKI group, but a significant decrease was observed already immediately post-op, compared to the pre-op value $(p<0.0001)$.

Serum NGAL levels were higher in the AKI group compared to the no-AKI group $(p<0.05)$ at every time point, except pre-op. Within the AKI group, a significant increase in NGAL from the pre-op value becomes already apparent post-op ( $p=0.0003)$. However, a significant increase is also seen in the no-AKI group, already present at the post-op day compared to the pre-op value $(p<$ $0.0001)$.

Serum CysC levels were significantly higher in the AKI group than the no-AKI group and this for every time point (pre-op $p=0.010$, post-op $p=0.005$, and all later times $p<0.0001)$. CysC levels increased gradually over time in 

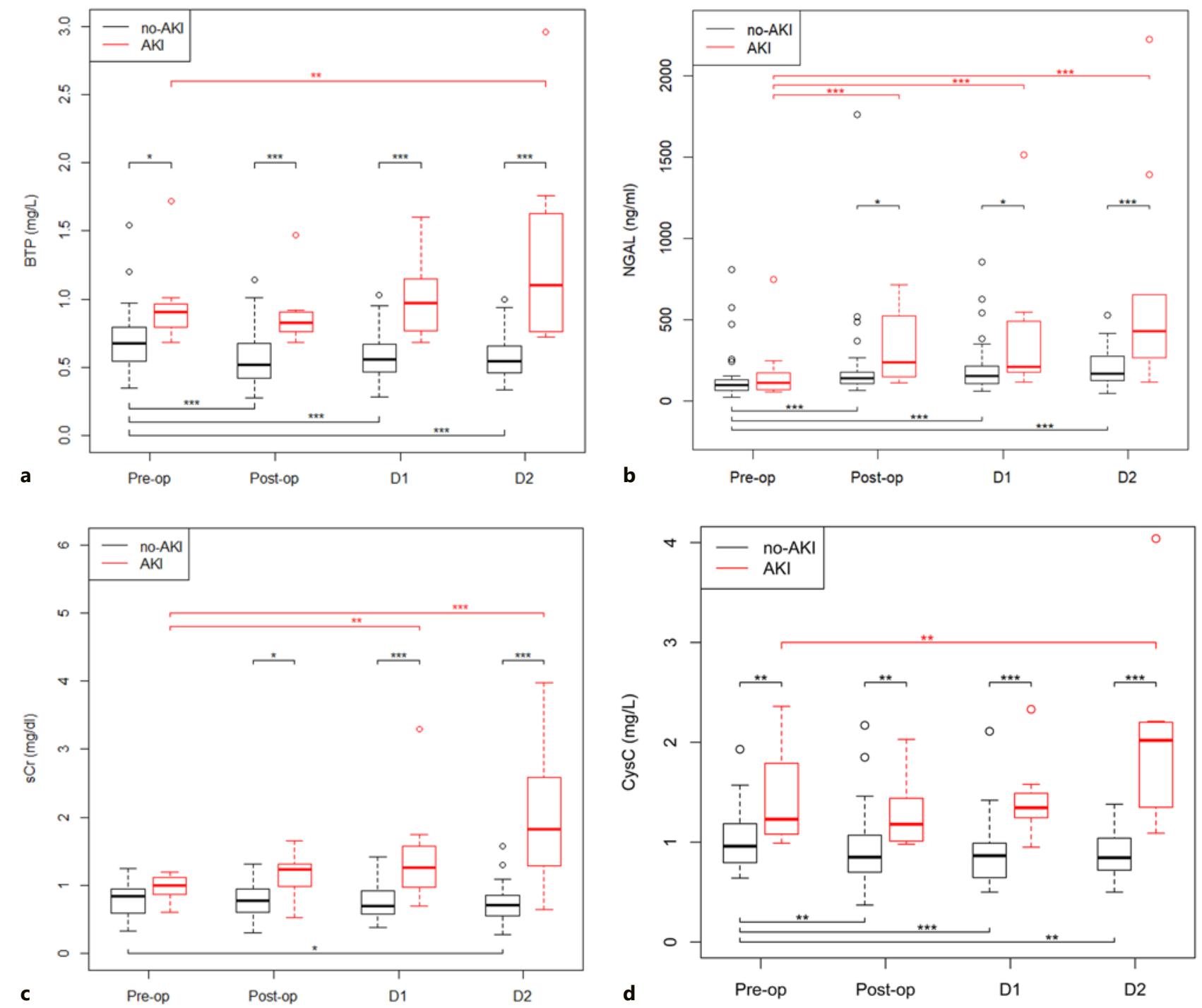

Fig. 1. Time course of BTP, NGAL, CysC, and $\mathrm{sCr}$ in the AKI and no-AKI groups. a shows the time course of BTP; $\mathbf{b}$ shows the time course of NGAL, $\mathbf{c}$ shows the time course of SCr, and $\mathbf{d}$ shows the time course of CysC. Significant between-group differences or significant differences from the pre-op value are given. ${ }^{*} p$ value $<0.05$;

the AKI group, reaching a statistically significant increase at day 2 compared to the pre-op values $(p=0.003)$. In contrast, no increase was seen in the no-AKI group, but a significant decrease was observed already immediately postop, compared to the pre-op value $(p=0.010)$.

For sCr, a gradual increase in $\mathrm{sCr}$ was seen post-op in patients who developed AKI compared to the no-AKI

${ }^{* *} p$ value $<0.01 ;{ }^{* * *} p$ value $<0.001 ; B T P$, beta-trace protein; NGAL, neutrophil gelatinase-associated lipocalin; sCr, serum $\mathrm{Cr}$; CysC, cystatin C; pre-op, preoperative; post-op, postoperative; D1, first postoperative day; D2, second postoperative day; AKI, acute kidney injury; no-AKI, no acute kidney injury.

group $(p<0.05)$, as reflected in the definition of AKI. This increase was statistically significant from D1 when comparing to the pre-op value of sCr within the AKI group ( $p$ value for $\mathrm{D} 1=0.001$; $p$ value for $\mathrm{D} 2<0.0001$ ). In the no-AKI group, $\mathrm{sCr}$ decreases, reaching statistical significance from D2 compared to pre-op value ( $p=$ $0.013)$. 

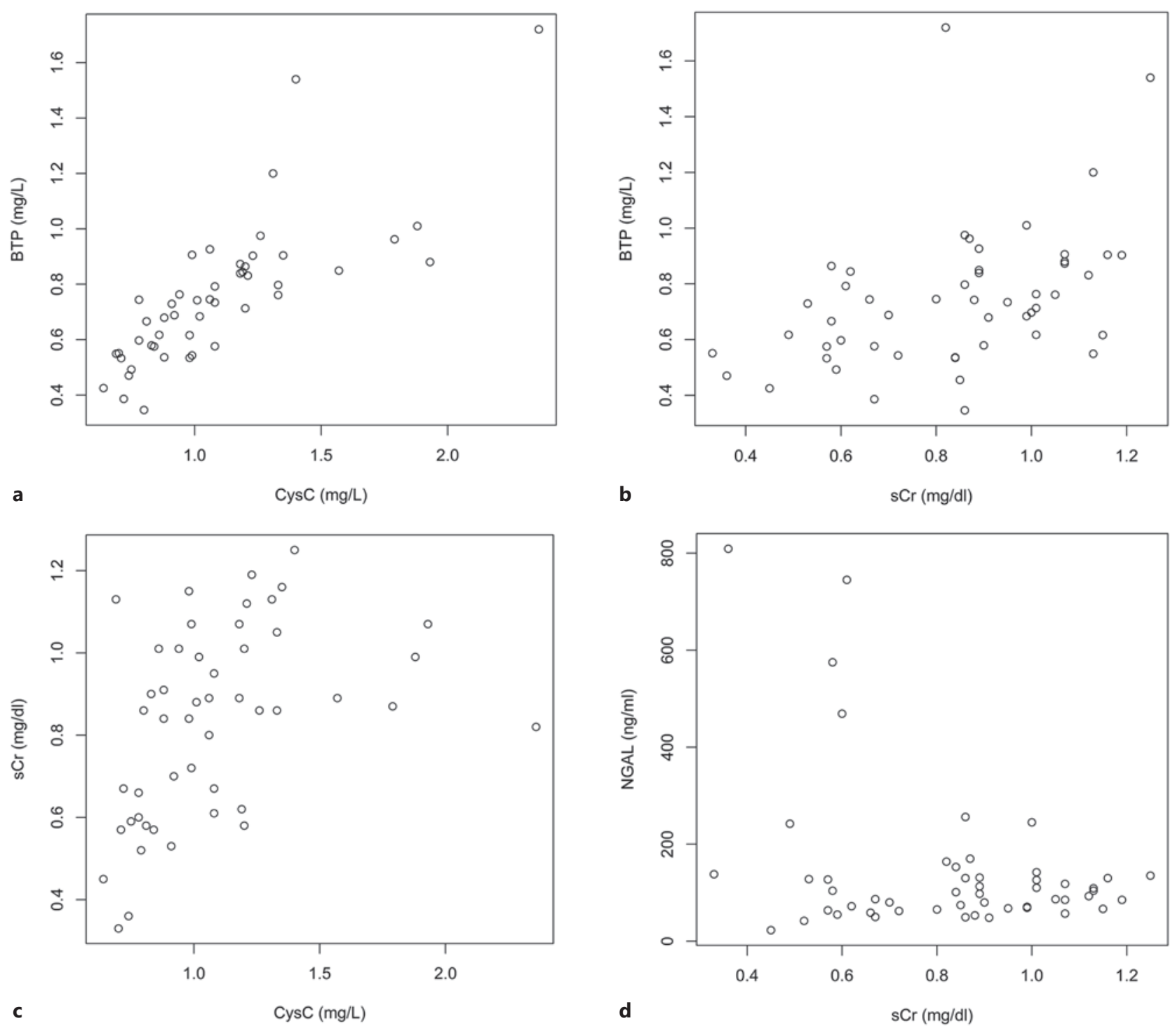

Fig. 2. Correlation between the pre-op BTP, CysC, NGAL, and SCr levels. a shows the correlation of pre-op BTP with pre-op CysC; b shows the correlation of pre-op BTP with pre-op SCr; c shows the correlation of pre-op CysC with pre-op SCr; and $\mathbf{d}$ shows the

correlation of pre-op NGAL with pre-op SCr. BTP, beta-trace protein; NGAL, neutrophil gelatinase-associated lipocalin; sCr, serum Cr; CysC, cystatin C.

Correlation between Beta-Trace Protein, Cystatin C, Neutrophil Gelatinase-Associated Lipocalin, and Serum $\mathrm{Cr}$

A strong positive correlation was found between preop serum levels of BTP and CysC ( $r=0.844, p=<0.0001)$. To a lesser extent, a positive correlation was seen between the pre-op serum levels of $\mathrm{Cr}$ and $\mathrm{CysC}(r=0.543, p<$ $0.0001)$ and between pre-op Cr and BTP $(r=0.490, p=$
$0.0003)$. No correlation was detected between pre-op serum levels of NGAL and $\operatorname{sCr}(r=0.038, p=0.787)$ (Fig. 2).

Beta-Trace Protein, Neutrophil Gelatinase-Associated Lipocalin, Cystatin C, Serum Cr, and Prediction of Acute Kidney Injury

ROC analysis for the detection of AKI within 2 days after surgery showed a great accuracy for BTP and CysC 


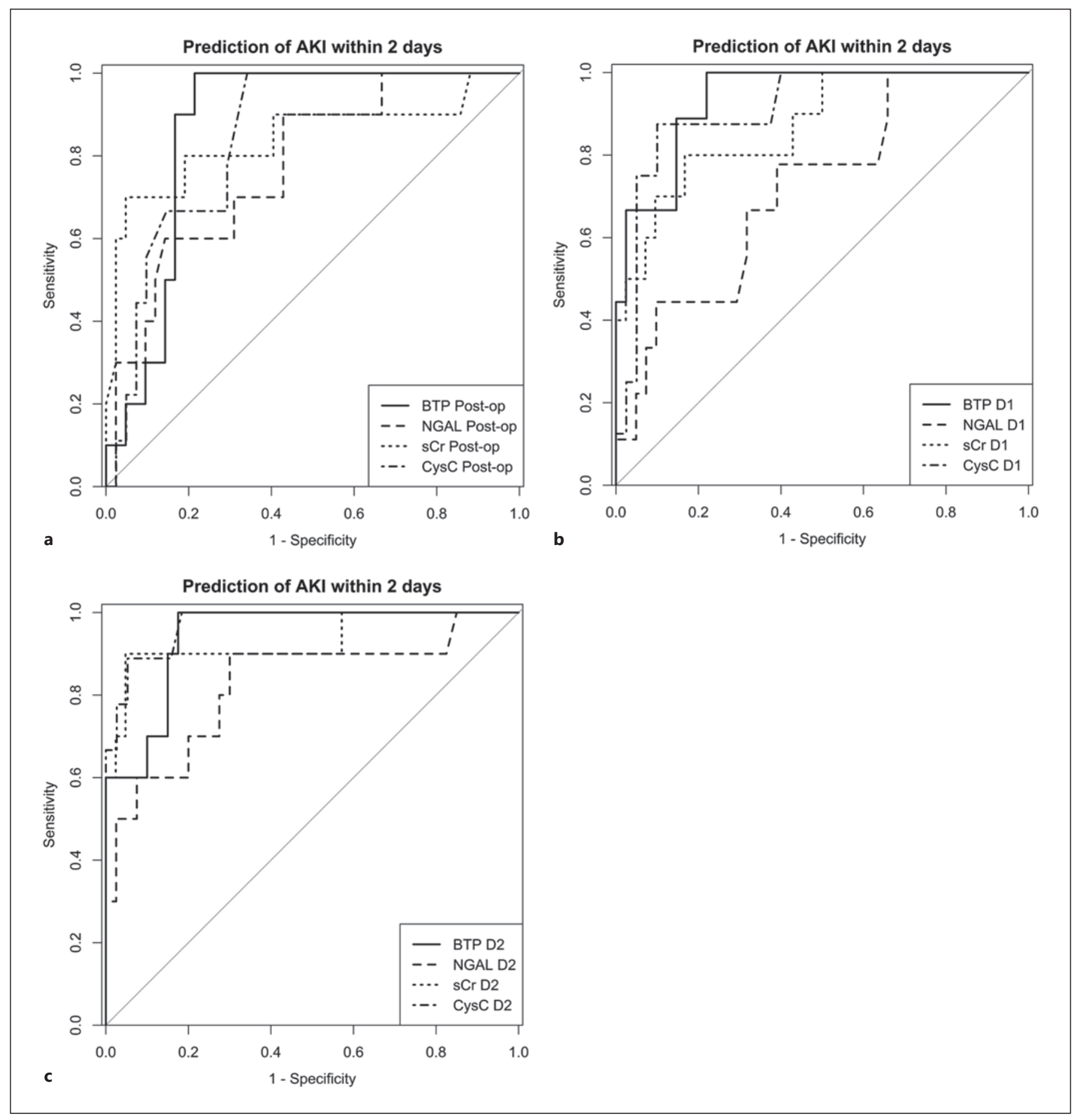

Fig. 3. ROC analysis for the prediction of AKI within 2 days after surgery using BTP, NGAL, CysC, and sCr values. a shows the ROC curve for prediction of AKI within 2 days after surgery for post-op values of BTP, NGAL, CysC, and sCr; $\mathbf{b}$ shows the ROC curve for prediction of AKI within 2 days after surgery for day 1 values of $\mathrm{BTP}, \mathrm{NGAL}, \mathrm{CysC}$, and sCr; $\mathbf{c}$ shows the ROC curve for prediction of AKI within 2 days after surgery for day 2 values of BTP, NGAL, CysC, and sCr. BTP, beta-trace protein; NGAL, neutrophil gelatinase-associated lipocalin; sCr, serum Cr; CysC, cystatin C; postop, postoperative; AKI, acute kidney injury; no-AKI, no acute kidney injury. 
Table 2. AUC for prediction of AKI within 2 days after surgery for BTP, NGAL, CysC, and sCr at different time points

\begin{tabular}{llll}
\hline Comparison between & AUC (A) & AUC (B) & $\begin{array}{l}\text { Adjusted } \\
p \text { value }\end{array}$ \\
\hline Post-op & & & \\
BTP (A) and NGAL (B) & 0.869 & 0.775 & 1 \\
NGAL (A) and sCr (B) & 0.775 & 0.840 & 1 \\
BTP (A) and sCr (B) & 0.869 & 0.840 & 1 \\
BTP (A) and CysC (B) & 0.870 & 0.848 & 1 \\
CysC (A) and NGAL (B) & 0.848 & 0.771 & 1 \\
CysC (A) and sCr (B) & 0.848 & 0.821 & 1 \\
\hline First post-op day (D1) & & & \\
BTP (A) and NGAL (B) & 0.938 & 0.718 & $\mathbf{0 . 0 2 7}$ \\
NGAL (A) and sCr (B) & 0.718 & 0.854 & 0.827 \\
BTP (A) and sCr (B) & 0.938 & 0.854 & 0.827 \\
BTP (A) and CysC (B) & 0.928 & 0.911 & 1 \\
CysC (A) and NGAL (B) & 0.911 & 0.725 & 0.176 \\
CysC (A) and sCr (B) & 0.911 & 0.856 & 1 \\
\hline Second post-op day (D) & & & \\
BTP (A) and NGAL (B) & 0.942 & 0.826 & 0.467 \\
NGAL (A) and sCr (B) & 0.826 & 0.940 & 0.131 \\
BTP (A) and sCr (B) & 0.942 & 0.940 & 1 \\
BTP (A) and CysC (B) & 0.944 & 0.972 & 0.799 \\
CysC (A) and NGAL (B) & 0.972 & 0.826 & 0.463 \\
CysC (A) and sCr (B) & 0.972 & 0.930 & 1 \\
\hline
\end{tabular}

Statistically significant values are reported in bold. AUC, area under the curve; BTP, beta-trace protein; NGAL, neutrophil gelatinase-associated lipocalin; CysC, cystatin $\mathrm{C}$; sCr, serum $\mathrm{Cr}$; AKI, acute kidney injury.

(Fig. 3). The area under the curve (AUC) for BTP post-op; D1; and D2 was, respectively, $0.869 \pm 0.049 ; 0.938 \pm 0.035$; and $0.943 \pm 0.032$ (Table 2). The AUC for CysC post-op, $\mathrm{D} 1$; and D2 was, respectively, $0.848 \pm 0.058 ; 0.911 \pm 0.053$; and $0.972 \pm 0.022$. The discriminative power of BTP measured on the first post-op day was superior to NGAL to detect AKI (adjusted $p$ value $=0.027$ ). There was no significant difference between the AUCs for BTP and CysC; and this for every time point (post-op adjusted $p$ value $=$ 1 ; D1 adjusted $p$ value $=1$; and D2 adjusted $p$ value $=$ 0.799). Also, CysC was not superior to NGAL on the first post-op day to detect AKI (adjusted $p$ value $=0.176$ ).

\section{Discussion}

This study was designed to evaluate the value of serum BTP in the diagnosis of AKI in an ICU setting, with surgery being a well-defined risk factor in time. Several find- ings emerge from this study. First, at every studied time point, serum BTP levels are higher in patients developing AKI than patients not developing AKI, which likely corresponds with a decreased renal clearance. Second, serum $\mathrm{BTP}$ rises early in the course of AKI, and its discriminative power on the first post-op day to detect AKI proves to be superior to that of NGAL.

In this study, about $20 \%$ of the patients developed AKI, defined as an increase of SCr to $\geq 1$.5-fold from baseline within 2 days after surgery. This is lower than the reported incidence in previous studies, probably reflecting the heterogeneity of the AKI definitions used in the literature (based on SCr and/or urine output), better perioperative care nowadays and short follow-up (2 days). Consistent with findings of other studies in similar populations, patients with AKI were generally older [19] and had a longer length of ICU stay $[8,19]$, compared to the patients without AKI. The higher age of patients in the AKI group might reflect the higher susceptibility of older patients to AKI. So far as we know, BTP values itself are not influenced by age [24]. In our study, when correcting for age in the mixed model for BTP, age appeared not to be significant in this model $(p=0.5003)$ and correcting for age did not have an impact on any of the reported results (estimates and $p$ values).

Although not significant in this small study population, patients with AKI tend to have a higher SOFA score than patients without AKI, which also is in line with findings in the literature $[8,19]$. We investigated the difference in AKI in patients with elective versus emergency surgery but could not detect any statistical difference (Fisher's exact test). However, we need to be careful to interpret these results, as the number of patients per group was very small (6 patients vs. 3 patients).

In patients developing AKI by day 2, pre-op BTP and CysC values, but not sCr and NGAL values, were significantly higher than the no-AKI group. This finding is consistent with a study of Saydam et al. [22] were AKI was evaluated after cardiac surgery. The early increase of BTP and $\mathrm{CysC}$ might reflect already some degree of kidney dysfunction prior to surgery, possibly induced by the medical condition necessitating the surgery. The absence of pre-op rise in $\mathrm{sCr}$ can be explained by tubular excretion of sCr when GFR decreases $[1,5,7,13-15]$. The absence of pre-op rise in NGAL levels in both groups is puzzling, as other groups observed an increase in NGAL 12-24 h before a rise in $\mathrm{sCr}$ in $\mathrm{AKI}[5,7,8,11-14,17-20]$. Different explanations may underly this observation. First, in contrast with BTP and CysC, NGAL is a rather specific marker of tubular injury $[8,11-14,16,17,19,37]$ and will 
not rise with only decreasing GFR. Second, the sensitivity of NGAL could be lower than $\mathrm{sCr}$ and BTP [40]. Third, NGAL is known to increase with systemic inflammation, possibly attenuating the difference pre-op between the AKI and no-AKI groups (as we see increasing NGAL levels over time in both groups) $[1,11]$.

The evolution of BTP over time was significantly different in the AKI versus no-AKI group. There is a small decrease of BTP immediate after surgery in both groups (only reaching statistical significance in the no-AKI group), followed by a steady increase in BTP levels in the AKI group while decreasing significantly in the no-AKI group. This early decrease of BTP immediately after surgery might reflect hemodilution [29, 41] (cfr. albumin/hematocrit drop - see online suppl. Fig. 1) or a decreased BTP production as a consequence of anesthesia, but further research is warranted before any conclusion can be drawn.

The discriminative power of BTP measured on the first post-op day was superior to NGAL to detect AKI. This is in line with another study where NGAL failed to demonstrate any true benefit in diagnosing AKI [42]. A possible explanation is that NGAL is elevated in an inflammatory state, attenuating its accuracy to detect AKI [42].

In our study, we could not detect a superiority of CysC to NGAL (as we found for BTP), nor was there a significant difference between CysC and the other markers, for detecting AKI within two days after surgery. This is in contrast with a recent published study by Saydam et al. [22]. In this study, the authors found that pre-op CysC, compared to pre-op sCr, had better results in predicting AKI compared to pre-op BTP and NGAL in comparison with pre-op sCr. It is difficult to compare both studies as Saydam et al. [22] only investigated the pre-op value of CysC in comparison with pre-op sCr to detect AKI. In our study, we did not use the pre-op values for the evaluation of the prediction of AKI as the surgery itself would be the risk factor for AKI. Therefore, we compared the post-op, D1, and D2 values of our markers and their accuracy for detection of AKI after surgery.

Another puzzling finding in this study is the rise of BTP in the no-AKI group post-op. This is in contrast with our study where we found declining levels of BTP post-op in the no-AKI groups. We do point out that a different BTP assay was used (ELISA vs. nephelometry), which precludes direct comparison of the results obtained in both studies [24]. Whether there is an effect of the anesthetics or the pre-op procedure itself (cardiopulmonary bypass) remains to be elucidated.

BTP is a promising biomarker and easy to measure since the technique (nephelometry) is not expensive and straight- forward. Moreover, technical variability is acceptable and, when reported, is consistently below $6.5 \%[24,30]$. Also, BTP measurements seem to be robust to a single freezethaw cycle [43]. In a study of Van Craenenbroeck et al. [36], an excellent reproducibility over time was found for both $\mathrm{HD}$ and $\mathrm{PD}$ patients with intra-class correlation coefficient of $0.96(0.93-0.99)$ and $0.92(0.86-0.99)$, respectively.

This study has several limitations. First, in the absence of a gold standard for diagnosing AKI, a definition based on only sCr was used. As already stated, $\mathrm{sCr}$ has several drawbacks, including the relative insensitivity to detect early AKI as it rises slowly after renal injury. However, since sCr was used for the definition of AKI, it is actually possible that the potential of BTP to detect AKI is even better than reported here. Second, a rather small sample size of a heterogenous ICU population was studied, which makes it impossible to draw conclusions about BTP as a marker of severity of kidney damage. Therefore, further studies with a larger sample size and more frequent BTP measurements are needed.

In conclusion, this is the one of the first studies showing an increase in serum BTP in post-op AKI in a real-life clinical ICU setting. These pilot data corroborate the hypothesis that serum BTP could be a new, inexpensive diagnostic tool for AKI, with superiority to NGAL. Before implementation can be expected, results should be validated in larger prospective studies.

\section{Acknowledgements}

The authors would like to thank Petra Vertongen and Erik Snelders for their logistical support.

\section{Statement of Ethics}

The Antwerp University Hospital's Institutional Ethical Review Board granted permission for this trial (EC 17/20/240) with waiving of informed consent as all samples were taken from the "left over" serum bank of the central lab. For all patients admitted at the ICU of the Antwerp University Hospital, patients and relatives are informed in a brochure upon admission that anonymized data and leftover samples might be used for research objectives. The principle of opt-out consent was used, but no patient disagreed afterward with participation in the trial.

\section{Conflict of Interest Statement}

The authors declare no conflicts of interest. The results presented in this paper have not been published previously in whole or part, except in abstract form (57th ERA-EDTA Congress, 2019). 


\section{Funding Sources}

A.H. Van Craenenbroeck was supported by an ERA-EDTA Short Term Fellowship.

\section{Author Contributions}

K.L., N.V.R., K.G., W.V., P.J., and A.H.V.C. were responsible for data acquisition. K.L., N.V.R., E.R., P.J., and A.H.V.C. analyzed and interpreted the data. K.L. wrote the draft of the manuscript, and N.V.R., E.R., K.G., M.C., P.J., W.V., and A.H.V.C. contributed to the final manuscript and approved the final version.

\section{References}

1 Park JT. Postoperative acute kidney injury. Korean J Anesthesiol. 2017;70(3):258-66.

2 Nonaka T, Kimura N, Hori D, Sasabuchi Y, Nakano M, Yuri K, et al. Predictors of acute kidney injury following elective open and endovascular aortic repair for abdominal aortic aneurysm. Ann Vasc Dis. 2018;11(3):298305.

3 Romagnoli S, Ricci Z, Ronco C. Perioperative acute kidney injury: prevention, early recognition, and supportive measures. Nephron. 2018;140(2):105-10.

4 Yousefshahi F, Fakhre Yasseri AM, Barkhordari K, Amini M, Salehi Omran A, Rezaei Hemami $\mathrm{M}$, et al. Incidence and complications of acute kidney injury following coronary artery bypass graft: a retrospective cohort study. Iran J Kidney Dis. 2015;9(2):113-8.

5 Jain V, Mehta Y, Gupta A, Sharma R, Raizada A, Trehan N. The role of neutrophil gelatinase-associated lipocalin in predicting acute kidney injury in patients undergoing offpump coronary artery bypass graft: a pilot study. Ann Card Anaesth. 2016;19(2):225-30.

6 Metzger J, Mullen W, Husi H, Stalmach A, Herget-Rosenthal S, Groesdonk HV, et al. Acute kidney injury prediction in cardiac surgery patients by a urinary peptide pattern: a case-control validation study. Crit Care. 2016; 20(1):157.

7 Mosa OF. Prognostic significance of serum NGAL and troponin I against acute kidney injury in Egyptian ICU patients after open heart surgery: a pilot study. Kidney Dis. 2018;4(4): 246-54.

8 de Geus HR, Bakker J, Lesaffre EM, le Noble JL. Neutrophil gelatinase-associated lipocalin at ICU admission predicts for acute kidney injury in adult patients. Am J Respir Crit Care Med. 2011;183(7):907-14.

9 Huen SC, Parikh CR. Molecular phenotyping of clinical AKI with novel urinary biomarkers. Am J Physiol Renal Physiol. 2015;309(5): F406-13.

10 Mehta RL, Kellum JA, Shah SV, Molitoris BA, Ronco C, Warnock DG, et al. Acute kidney injury network: report of an initiative to improve outcomes in acute kidney injury. Crit Care. 2007;11(2):R31.

11 Pozzoli S, Simonini M, Manunta P. Predicting acute kidney injury: current status and future challenges. J Nephrol. 2018;31(2):209-23.
12 Schrezenmeier EV, Barasch J, Budde K, Westhoff T, Schmidt-Ott KM. Biomarkers in acute kidney injury: pathophysiological basis and clinical performance. Acta Physiol. 2017; 219(3):554-72.

13 Moledina DG, Parikh CR. Phenotyping of acute kidney injury: beyond serum creatinine. Semin Nephrol. 2018;38(1):3-11.

14 de Geus HR, Betjes MG, Bakker J. Biomarkers for the prediction of acute kidney injury: a narrative review on current status and future challenges. Clin Kidney J. 2012;5(2):102-8.

15 Khwaja A. KDIGO clinical practice guidelines for acute kidney injury. Nephron Clin Pract. 2012;120(4):c179-84.

16 Haase-Fielitz A, Haase M, Devarajan P. Neutrophil gelatinase-associated lipocalin as a biomarker of acute kidney injury: a critical evaluation of current status. Ann Clin Biochem. 2014;51(Pt 3):335-51.

17 Helanova K, Spinar J, Parenica J. Diagnostic and prognostic utility of neutrophil gelatinase-associated lipocalin (NGAL) in patients with cardiovascular diseases: review. Kidney Blood Press Res. 2014;39(6):623-9.

18 Firu SG, Streba CT, Firu D, Tache DE, Rogoveanu I. Neutrophil gelatinase associated lipocalin (NGAL): a biomarker of renal dysfunction in patients with liver cirrhosis: do we have enough proof? J Med Life. 2015;8(Spec Issue):15-20.

19 Cruz DN, de Cal M, Garzotto F, Perazella MA, Lentini P, Corradi V, et al. Plasma neutrophil gelatinase-associated lipocalin is an early biomarker for acute kidney injury in an adult ICU population. Intensive Care Med. 2010; 36(3):444-51.

20 Wang C, Zhang J, Han J, Yang Q, Liu J, Liang B. The level of urinary IL-18 in acute kidney injury after cardiopulmonary bypass. Exp Ther Med. 2017;14(6):6047-51.

21 Chen HH. $\beta$-trace protein versus cystatin C: which is a better surrogate marker of renal function versus prognostic indicator in cardiovascular diseases? J Am Coll Cardiol. 2011; 57(7):859-60

22 Saydam O, Türkmen E, Portakal O, Arıcı M, Doğan R, Demircin M, et al. Emerging biomarker for predicting acute kidney injury after cardiac surgery: cystatin C. Turk J Med Sci. 2018;48(6):1096-103.

23 Ferguson MA, Waikar SS. Established and emerging markers of kidney function. Clin Chem. 2012;58(4):680-9.
24 White CA, Ghazan-Shahi S, Adams MA. $\beta$-trace protein: a marker of GFR and other biological pathways. Am J Kidney Dis. 2015; 65(1):131-46.

25 Inker LA, Tighiouart H, Coresh J, Foster MC, Anderson AH, Beck GJ, et al. GFR estimation using $\beta$-trace protein and $\beta 2$-microglobulin in CKD. Am J Kidney Dis. 2016;67(1):40-8.

26 Poge U, Gerhardt TM, Stoffel-Wagner B, Palmedo H, Klehr HU, Sauerbruch T, et al. $\beta$-trace protein is an alternative marker for glomerular filtration rate in renal transplantation patients. Clin Chem. 2005;51(8):1531-3.

27 Priem F, Althaus H, Birnbaum M, Sinha P, Conradt HS, Jung K. Beta-trace protein in serum: a new marker of glomerular filtration rate in the creatinine-blind range. Clin Chem. 1999;45(4):567-8.

28 Shafi T, Pluznick JL. Renal handling of betatrace protein: interpreting the evidence. Am J Kidney Dis. 2015;65(6):967.

29 Clausen J. Proteins in normal cerebrospinal fluid not found in serum. Proc Soc Exp Biol Med. 1961;107:170-2.

30 Donadio C. Serum and urinary markers of early impairment of GFR in chronic kidney disease patients: diagnostic accuracy of urinary beta-trace protein. Am J Physiol Renal Physiol. 2010;299(6):F1407-23.

31 Poge U, Gerhardt T, Woitas RP. Estimation of glomerular filtration rate by use of beta-trace protein. Clin Chem. 2008;54(8):1403-5.

32 Shafi T, Michels WM, Levey AS, Inker LA, Dekker FW, Krediet RT, et al. Estimating residual kidney function in dialysis patients without urine collection. Kidney Int. 2016; 89(5):1099-110.

33 White CA, Akbari A, Doucette S, Fergusson D, Hussain N, Dinh L, et al. A novel equation to estimate glomerular filtration rate using beta-trace protein. Clin Chem. 2007;53(11): 1965-8.

34 Gerhardt T, Pöge U, Stoffel-Wagner B, Klein B, Klehr HU, Sauerbruch T, et al. Serum levels of beta-trace protein and its association to diuresis in haemodialysis patients. Nephrol Dial Transplant. 2008;23(1):309-14.

35 Shafi T, Parekh RS, Jaar BG, Plantinga LC, Oberai PC, Eckfeldt JH, et al. Serum $\beta$-trace protein and risk of mortality in incident hemodialysis patients. Clin J Am Soc Nephrol. 2012;7(9):1435-45. 
36 van Craenenbroeck AH, Bragfors-Helin AC, Qureshi AR, Lindholm B, Sjöberg B, Anderstam B, et al. Plasma beta-trace protein as a marker of residual renal function: the effect of different hemodialysis modalities and intraindividual variability over time. Kidney Blood Press Res. 2017;42(5):877-85.

$37 \mathrm{Wu} \mathrm{T,} \mathrm{Fu} \mathrm{Y,} \mathrm{Brekken} \mathrm{D,} \mathrm{Yan} \mathrm{M,} \mathrm{Zhou} \mathrm{XJ,}$ Vanarsa $\mathrm{K}$, et al. Urine proteome scans uncover total urinary protease, prostaglandin $\mathrm{D}$ synthase, serum amyloid $\mathrm{P}$, and superoxide dismutase as potential markers of lupus nephritis. J Immunol. 2010;184(4):2183-93.

38 de Grooth HJ, Geenen IL, Girbes AR, Vincent JL, Parienti JJ, Oudemans-van Straaten HM. SOFA and mortality endpoints in randomized controlled trials: a systematic review and meta-regression analysis. Crit Care. 2017; 21(1):38.
39 Zimmerman JE, Kramer AA, McNair DS, Malila FM. Acute physiology and chronic health evaluation (APACHE) IV: hospital mortality assessment for today's critically ill patients. Crit Care Med. 2006;34(5):1297310.

40 Perry TE, Muehlschlegel JD, Liu KY, Fox AA, Collard CD, Shernan SK, et al. Plasma neutrophil gelatinase-associated lipocalin and acute postoperative kidney injury in adult cardiac surgical patients. Anesth Analg. 2010;110(6): 1541-7.
41 White CA, Akbari A, Doucette S, Fergusson D, Ramsay T, Hussain N, et al. Effect of clinical variables and immunosuppression on serum cystatin $\mathrm{C}$ and beta-trace protein in kidney transplant recipients. Am J Kidney Dis. 2009;54(5):922-30.

42 Martensson J, Bell M, Oldner A, Xu S, Venge P, Martling CR. Neutrophil gelatinase-associated lipocalin in adult septic patients with and without acute kidney injury. Intensive Care Med. 2010;36(8):1333-40.

43 Juraschek SP, Coresh J, Inker LA, Rynders GP, Eckfeldt JH, Selvin E. The effects of freeze-thaw on $\beta$-trace protein and $\beta 2$ microglobulin assays after long-term sample storage. Clin Biochem. 2012;45(9):694-6. 